\title{
Evaluation of stemness marker expression in bovine ovarian granulosa cells
}

\author{
Son Nghia Hoang ${ }^{1}$, Chi Nguyen Quynh Ho ${ }^{1}$, Thao Thi Phuong Nguyen ${ }^{1}$, Chung Chinh Doan ${ }^{1}$, \\ Diem Hong Tran', Long Thanh Le ${ }^{1, \S}$
${ }^{1}$ Animal Biotechnology Department, Institute of Tropical Biology, Vietnam Academy of Science and Technology, Ho Chi Minh City, Vietnam.
${ }^{2}$ Department of Internal Medicine - Cardiology, UT Southwestern Medical Center, Dallas, TX.

\begin{abstract}
The objective of this study was to assess the stemness marker expressions (Oct4, Nanog, and Sox2) of granulosa cells (GCs) collected from bovine ovarian follicles and in vitro expansion. The single bovine ovarian follicles were isolated and categorized into 4 groups according to their diameter including group $\mathrm{A}$ ( $<2 \mathrm{~mm}$ ), group B (2-3 mm), group C (3-4 mm), and group $\mathrm{D}(>4 \mathrm{~mm})$. Quantitative reverse transcriptase polymerase chain reaction (qRT-PCR) and immunostaining were applied to evaluate the stemness marker expression of bovine GCs from ovarian follicles. We also estimated the stemness marker transcript expressions of GCs during in vitro expression by qRT-PCR. qRT-PCR analysis demonstrated that fresh GCs from bovine ovarian follicles expressed the stemness markers (Oct4, Nanog, Sox2). These markers were down-regulated during antral stage follicular development. We also estimated stemness marker transcript expressions of GCs which were isolated and in vitro expanded from ovarian follicles of group A. The qRT-PCR results showed that Oct4 and Sox2 transcript expressions were reduced during in vitro expansion while Nanog transcript was not expressed.
\end{abstract}

Keywords: granulosa cells, ovarian follicles, transcript expression, stemness markers.

\section{Introduction}

The mammalian ovarian follicle development is begun from the primordial follicle, which contains an immature small egg and a single undifferentiated granulosa cell layer (Gougeon, 1996). This cell layer is surrounded by a follicular wall (Gougeon, 1996). During follicular development, the surrounding GCs begin to undergo the process of maturation, the number of GCs increases and the basal membrane is enlarged (Rodgers et al., 1999; 2001). The rapid proliferation of GCs suggests that there is an involvement of stem cell basedmechanisms during folliculogenesis (Chronowska, 2012). Many studies have provided evidence that GCs carry the characteristics of stem cells (Chronowska, 2012). Stem cell properties can be displayed in GCs by lacking mitotic inhibition of transcription (Rodgers et al., 1999), the ability to divide without anchorage to a substratum in vitro (Potten et al., 1990; Copper, 1997). Following the study of Kossowska-Tomaszczuk on luteinizing GCs isolated from the ovarian follicles of infertile patients, OCT4, a typical stem cell marker, was expressed in long-term culture with the presence of the leukemiainhibiting factor (Kossowska-Tomaszczuk et al., 2009).

The potential properties of GCs have been characterized in human (Kossowska-Tomaszczuk et al., 2009; Parte et al., 2011; Varras et al., 2012), mouse (Bagheripour et al., 2017), porcine (Mattiolia et al., 2012; Oki et al., 2012). These studies characterized the stemness marker expression of GCs in developing follicles from specific stages. An evidence for ovarian granulosa stem cells in bovine follicles was firstly demonstrated with high activity of telomerase (Lavranos et al., 1999). However, the potential of bovine ovarian GCs has not been well described, especially the changes of stemness marker expression in follicular development. Thus, the current study was aimed to investigate the expression and changes of stemness markers (Oct4, Nanog, Sox2) in bovine GCs from developing follicles. The results of this study could contribute to understanding of potential of bovine GCs.

\section{Materials and Methods}

\section{Isolation and culture bovine granulosa cells}

Bovine ovaries were obtained from slaughter house and transported to the laboratory within 3 hours. They were kept in $0.9 \%$ normal saline. Single bovine follicles were collected by dissection under a stereomicroscope (Meiji, Japan).

To estimate the stemness transcript expression of fresh GCs, four bovine follicle groups were selected based on diameter including group A $(<2 \mathrm{~mm})$, group B $(2-3 \mathrm{~mm})$, group $\mathrm{C}(3-4 \mathrm{~mm})$, and group $\mathrm{D}(>4 \mathrm{~mm})$. Single bovine follicles were washed in Dulbecco's Modified Eagle Medium (DMEM, Gibco, Germany) supplemented with $10 \%$ fetal bovine serum (FBS, Gibco, Germany) and $1 \%$ antibiotic (penicillin/streptomycin, Gibco, Germany). The fresh GCs were collected from these above follicles by aspiration and the oocytecumulus complexes were removed. These fresh GCs were used for stemness transcript analysis by qRT-PCR.

To estimate the stemness transcript expression of GCs from in vitro expansion, the granulosa from group A were collected and cultured. Bovine GCs were aspirated and transferred to $\Phi 35 \mathrm{~mm}$ tissue culture dishes containing DMEM supplemented with $10 \%$ FBS and $1 \%$ penicillin/streptomycin. The oocyte-cumulus complexes were removed from collecting GCs. GCs were cultured at $37.5^{\circ} \mathrm{C}$ in a $5 \% \mathrm{CO}_{2}$ atmosphere. The GCs from passage 0 , passage 1 , and passage 4 were used for stemness transcript analysis by qRT-PCR. In this examination, the GCs of passage 0 were different from fresh GCs. They were isolated from follicle and primarily cultured for several days. After exhibiting $90 \%$ confluence, the primary cultured GCs of passage 0 were collected and used for transcript expression analysis. 


\section{Total RNA extraction}

The bovine GCs were harvested and washed twice with phosphate-buffered saline (PBS) (Gibco, Germany). Their total RNA was extracted using a Ribospin $^{\text {TM }}$ Total RNA Purification Kit (GeneAll Biotechnology, Korea), according to the manufacturer's instructions. The quality and quantity of the RNA sample were assessed using the spectrophotometer (NanoVue Plus Spectrophotometer, GE Healthcare Life Sciences, United States). RNA samples with a ratio of $\mathrm{A}_{260} / \mathrm{A}_{280}$ between 1.8 and 2.0 were aliquoted and used for qRT-PCR.

\section{Quantitative Real time RT-PCR}

PikoReal 96 Real-Time PCR System (Thermo
Scientific, United States) was used for qRT-PCR. qRTPCR reactions were performed with qPCR SyGreen 1Step Lo-ROX kit (Biosystem, England). qRT-PCR was conducted in $20 \mu \mathrm{l}$ for each reaction, including $1 \mu \mathrm{l}$ of total RNA, $2 \mu \mathrm{l}$ of primers (forward and reverse) (Table 1) (Sumer et al., 2011), $10 \mu \mathrm{l}$ Mix Ro-Lox, $1 \mu \mathrm{l}$ RTAse, and $6 \mu \mathrm{dH}_{2} \mathrm{O}$. The qRT-PCR reactions were performed by one cycle of $45^{\circ} \mathrm{C}$ for $15 \mathrm{~min}$, one cycle of $95^{\circ} \mathrm{C}$ for 2 min, 40 cycles of $95^{\circ} \mathrm{C}$ for $10 \mathrm{sec}, 62^{\circ} \mathrm{C}$ for $15 \mathrm{sec}$; and 71 cycles of $60^{\circ} \mathrm{C}$ for $30 \mathrm{sec}$. $\beta$-actin was used as internal control, the $2^{-\Delta \Delta \mathrm{Ct}}$ method was applied for $\mathrm{Ct}$ value analysis (Livak et al., 2001). PCR products were electrophoresed on a $1 \%$ agarose gel and stained with GelRed (Biotium, United States). The gel was exposed to UV light and the picture taken with a gel documentation system (Infinity, Vilber Lourmat, France).

Table 1. Primers were used in this study for qRT-PCR.

\begin{tabular}{llc}
\hline Gene & \multicolumn{1}{c}{ Primer $\left(5^{\prime}-3^{\prime}\right)$} & Product size $(\mathrm{bp})$ \\
\hline \multirow{2}{*}{ Oct4 } & F: GTTCTCTTTGAAAGGTGTTC & 313 \\
& R: ACACTCGGACCACGTCTTTC & \\
Nanog & F: GTGTTTGGTGACTCTCCTG & 307 \\
& R: GGGAATTGAAATACTTGACAG & \\
Sox-2 & F: CATCCACAGCAAATGACAGC & 251 \\
& R: TTTCTGCAAAGCTCCTACCG & \\
$\beta$-actin & F: GGAATCCTGTGGCATCCATGAAAC & 348 \\
\hline
\end{tabular}

\section{Immunostaining}

This assay was applied to assess the OCT4 and NANOG expression from fresh GCs. GCs were collected from bovine ovarian follicle of group A and cultured in $\Phi 35 \mathrm{~mm}$ tissue culture dish containing DMEM supplemented with $10 \%$ FBS and $1 \%$ penicillin/streptomycin. After $3 \mathrm{~h}, \mathrm{GCs}$ attached to dishes and were used for OCT4 and NANOG immunostaining assays. GCs were fixed with $4 \%$ paraformaldehyde in PBS (Nacalai, Japan) at room temperature for $30 \mathrm{~min}$, then permeabilized with $0.1 \%$ Triton X-100 in PBS (Merck, Germany) at room temperature for $30 \mathrm{~min}$. GCs were incubated with diluted primary antibodies overnight at $4{ }^{\circ} \mathrm{C}$ for OCT4 (sc-8629, Santa Cruz Biotechnology, United States) and NANOG (sc-30328, Santa Cruz Biotechnology, United States). GCs were incubated with donkey anti-goat IgGFITC (sc-2024, Santa Cruz Biotechnology, United States) at room temperature in the dark for an hour. GC nucleus was stained with 4',6-Diamidino-2phenylindole dihydrochloride (DAPI) (Sigma, United States) for $30 \mathrm{~min}$. The cells were washed with PBS three times in $5 \mathrm{~min}$ for each step. The stained GCs were observed under the fluorescent microscope to assess the OCT4 and NANOG expression.

\section{Statistical analysis}

Quantitative gene expression results were analyzed by Piko Real Software 2.2 software (Thermo Scientific, United States). $\beta$-actin was used as an internal control. Statistical analysis was performed by Sigma Plot 14 Software (Systat Software Inc., UK). The experiments were triplicated. The stemness transcript expressions were analyzed for statistical significance by one-way ANOVA where $\mathrm{P}<0.05$ was considered statistically significant.

\section{Results}

\section{Stemness marker expression of fresh GCs}

In this study, we assessed the stemness gene expression of GCs from bovine ovary follicles with different diameters and in vitro expansion. The qRTPCR showed that the transcript expression of stemness markers (Oct4, Nanog and Sox2) were detected in fresh GCs from ovarian follicles with the specific bands in electrophoresis (Fig. 1). These stemness gene expressions were down-regulated in GCs following the increase of bovine ovary follicle diameter. The Oct4, Nanog and Sox 2 transcript expression were highest in group A which was smaller than $2 \mathrm{~mm}$ in diameter. GCs of group B had a higher Oct4 and Sox2 transcript expressions than group $\mathrm{C}$ and $\mathrm{D}$, but there was no significant difference in Nanog expression between these groups. qRT-PCR also revealed a higher Oct4 transcript expression than Nanog and Sox 2 transcripts in group A follicles. During primary culture, the GCs attached to the culture dish in 3 hours. Immunohistochemistry showed that both OCT4 and NANOG markers were expressed in the nuclei and cytoplasm of GCs (Fig. 2). 

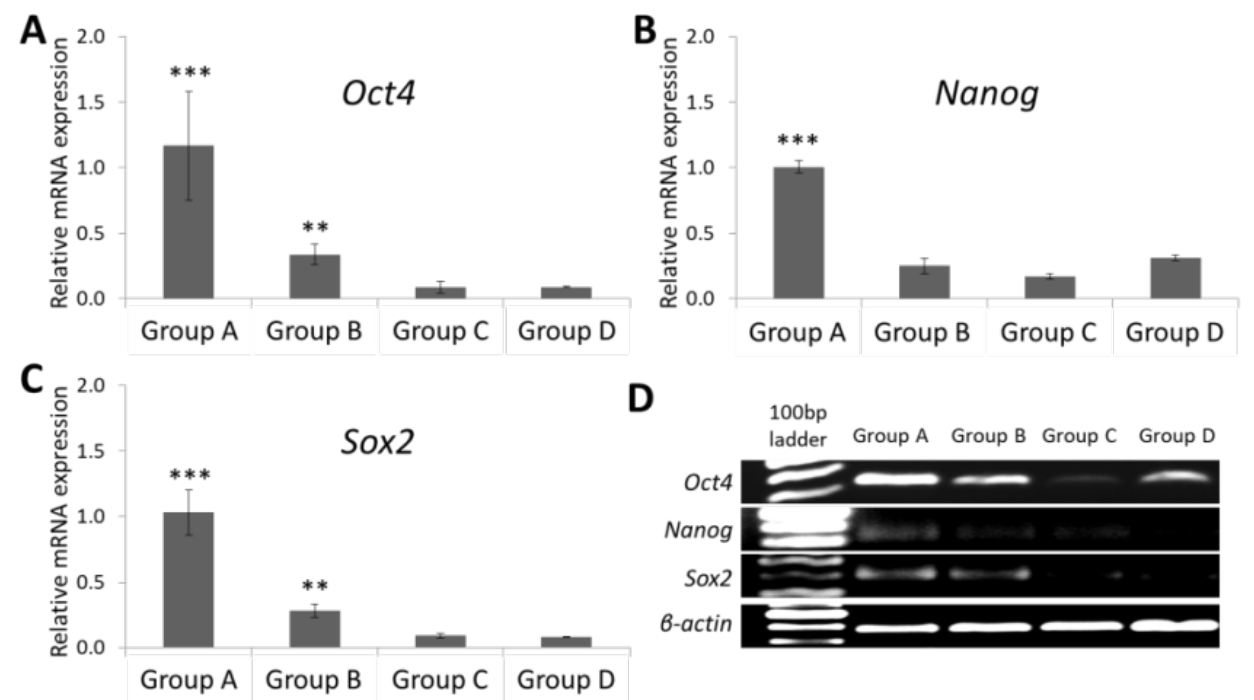

Figure 1. Quantitative Reverse transcriptase polymerase chain reaction analysis shows the expressions of stemness markers: (A), (B), (C) mRNA expression levels of Oct4, Nanog, and Sox2 (D) agarose gel electrophoresis and Gelred staining after qRT-PCR. ${ }^{* * *} \mathrm{P}<0.001$ (group A) vs. other groups, ${ }^{* *} \mathrm{P}<0.01$ (group B) vs. group C and group D.

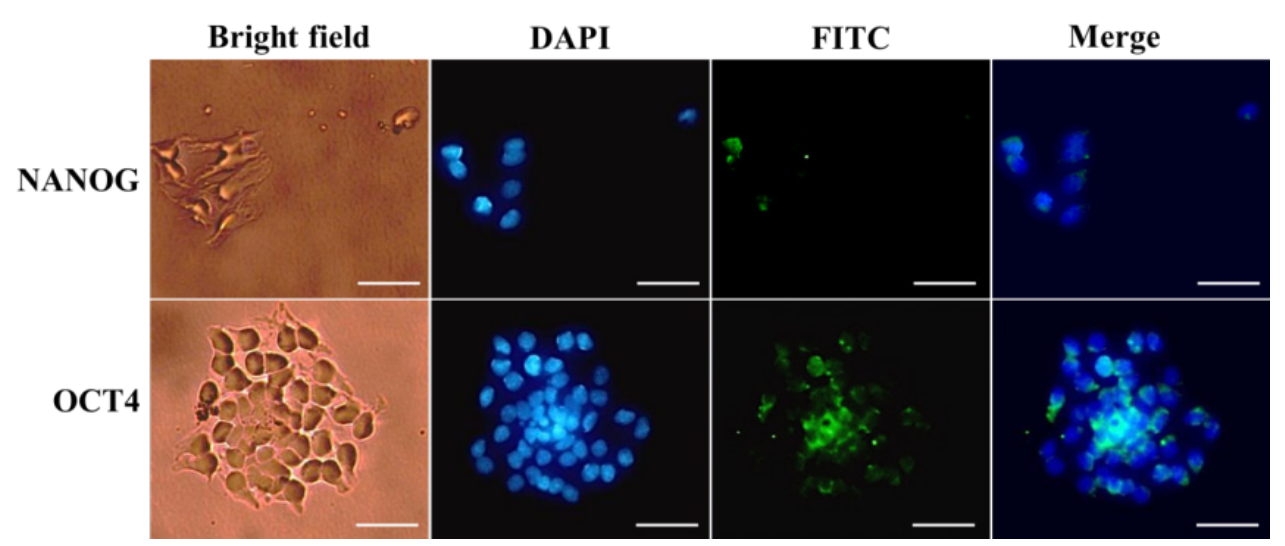

Figure 2. Immunofluorescence staining of GCs: OCT4 and NANOG expression were detected in fresh GCs from bovine ovarian follicle, which was smaller than $2 \mathrm{~mm}$ in diameter. Scale bar: $50 \mu \mathrm{m}$.

Stemness marker expression of GCs from in vitro expansion

The Group A follicles were used for GCs isolation and in vitro culture to estimate stemness marker expression. The results demonstrated that the Oct 4 and Sox 2 transcripts were determined in GCs in the in vitro expansion, however, these gene expressions were down-regulated from passage 1 to passage 4 (Fig. 3). The Oct4 transcript expression of GCs from passage 1 was higher than passage 4 , but there was no significant difference in Sox 2 transcript expression between the two passages.

In real time RT-PCR analysis, the $\mathrm{Ct}$ values were recorded when RFUs (relative fluorescence units) were higher than 100 (this is the threshold in PikoThermo Realtime System). The RFUs of Oct4 and Sox2 raised higher than 3500 and 1000 , respectively, while all RFUs of Nanog from P0, P1, and P4 were lower than 90. Thus, there was no Nanog transcript expression of bovine GCs from P0, P1, P4.

\section{Discussion}

Recently, the potential of ovarian somatic stem cells has been increasingly described. The ovaries have been proved to contain somatic stem cell population in ovarian epithelium layer (Parte et al., 2011), stromal layer and theca layer (Gong et al., 2010; Bui et al., 2014). One of the first study, which presented the potential of GCs was indicated by Kossowska-Tomaszczuk et al. (2009). They showed that GCs could in vitro differentiate into other cell types such as neurons, chondrocytes, and osteoblasts (Kossowska-Tomaszczuk et al., 2009).

In our study, the fresh GCs from bovine ovary follicles expressed stemness markers (Oct4, Nanog, and Sox2). This stemness transcripts of GCs were downregulated when follicle diameter increased. All of bovine ovarian follicles in this study were tertiary follicles (antral follicles), which are about 0.5 to $25 \mathrm{~mm}$ in diameter (Townson et al., 2012). The GCs are highly differentiated in the pre-ovulatory follicle in folliculogenesis (Li et al., 2012). It appears that differentiation is a cause of reduced expression of stemness markers. Therefore, the 
potential of granulosa stem-like cells could be decreased during antral stage follicular development. We also investigated the stemness marker expression during in vitro expansion, in which the Oct4 and Sox 2 transcript expression while Nanog transcript was not expressed. These results showed that bovine GCs progressively lost their original characteristics during in vitro expansion.

The other studies also indicated that the stemness markers were detected in GCs in different animals. Porcine GCs from growing and luteinizing follicles showed an expression of SOX2 and NANOG markers immediately after isolation while OCT4 expression was not detected (Mattiolia et al., 2012). These stemness markers expressions were maintained during the in vitro expansion of porcine GCs (Mattiolia et al., 2012). Additionally, the Oct4 mRNA was not expressed in the collected follicular-phase porcine GCs (Oki et al., 2012). However, the expression of human GC stemness markers was different from porcine. The OCT4 was expressed in the freshly collected luteinizing GCs and remained expressed in the luteinizing GCs throughout the culturing (Dzafic et al., 2013). Another study also showed that subpopulation of luteinized GCs in healthy human ovarian follicles expressed Oct4 transcripts (Varras et al., 2012). Our results are consistent with those above studies suggesting that mammalian ovary follicles contain GC populations, which exhibit their stemness properties. Moreover, the stemness marker expressions of granulosa stem-like cells are different from species, especially Oct4 and Nanog which are the most important regulators of stemness (Boyer et al., 2005).

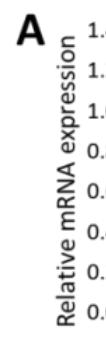


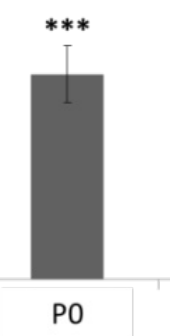

\section{Oct4}

B
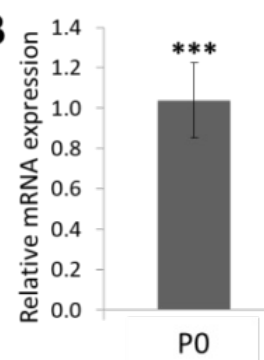



Sox 2

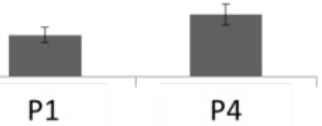

C



Figure 3. Quantitative Reverse transcriptase polymerase chain reaction analysis shows the expressions of stemness markers (A), (B) mRNA expression levels of Oct4 and Sox2 (C) granulosa cell morphology from passage 0 (P0), passage $1(\mathrm{P} 1)$, and passage $4(\mathrm{P} 4) .{ }^{* * *} \mathrm{P}<0.001(\mathrm{P} 0)$ vs. $\mathrm{P} 1$ and $\mathrm{P} 4,{ }^{* *} \mathrm{P}<0.01(\mathrm{P} 1)$ vs. $\mathrm{P} 4$.

\section{Acknowledgments}

This work was funded by Grant VAST02.03/1718 from Vietnam Academy of Science and Technology. All authors have reported no conflict of interest.

\section{References}

Bagheripour N, Zavareh S, Ghorbanian MT, Paylakhi SH, Mohebbi SR. 2017. Changes in the expression of OCT4 in mouse ovary during estrous cycle. Vet Res Forum, 8(1):43-48.

Boyer LA, Lee TI, Cole MF, Johnstone SE, Levine SS, Zucker JP, Guenther MG, Kumar RM, Murray HL, Jenner RG, Gifford DK, Melton DA, Jaenisch R, and Young RA. 2005. Core Transcriptional Regulatory Circuitry in Human Embryonic Stem Cells. Cell, 122(6):947-956.

Bui HT, Van Thuan N, Kwon DN, Choi YJ, Kang MH, Han JW, Kim T, Kim JH. 2014. Identification and characterization of putative stem cells in the adult pig ovary. Development, 141:2235-2244.
Chronowska E. 2012. Stem cell characteristics of ovarian granulose cells. Ann Anim Sci, 12(2):151-157.

Copper GM. 1997. The cell, a molecular approach. Oxford University Press, London. pp.604-608.

Dzafic E, Stimpfel M, Virant-Klun I. 2013. Plasticity of granulosa cells: on the crossroad of stemness and transdifferentiation potential. J Assist Reprod Genet, 30:1255-1261

Gong SP, Lee ST, Lee EJ, Kim DY, Lee G, Chi SG, Ryu BK, Lee CH, Yum KE, Lee HJ, Han JY, Tilly JL, Lim JM. 2010. Embryonic stem cell-like cells established by culture of adult ovarian cells in mice. Fertil Steril, 93:2594-2601.

Gougeon A. 1996. Regulation of ovarian follicular development in primates: facts and hypotheses. Endocr Rev, 17(2):121-155.

Kossowska-Tomaszczuk K, De Geyter C, De Geyter M, Martin I, Holzgreve W, Scherberich A, Zhang $H$. 2009. The multipotency of luteinizing granulosa cells collected from mature ovarian follicles. Stem Cells, 27:210-219.

Lavranos TC, Mathis JM, Latham SE, Kalionis B, 
Shay JW, and Rodgers RJ. 1999. Evidence for Ovarian Granulosa Stem Cells: Telomerase Activity and Localization of the Telomerase Ribonucleic Acid Component in Bovine Ovarian Follicles. Biol Reprod, 61:358-366.

Li H, Chian RC. 2012. Follicular Development and Oocyte Growth. Development of In Vitro Maturation for Human Oocytes. Springer, 37-57.

Livak KJ, Schmittgen TD. 2001. Analysis of relative gene expression data using Real-Time Quantitative PCR and the 2- $\Delta \Delta \mathrm{Ct}$ Method Methods, 25:402-408.

Mattioli M, Gloria A, Turriani M, Berardinelli P, Russo V, Nardinocchi D, Curini V, Baratta $M$, Martignani E, Barboni B. 2012. Osteo-regenerative potential of ovarian granulosa cells: An in vitro and in vivo study. Theriogenology, 77:1425-1437.

Oki Y, Ono H, Motohashi T, Sugiura N, Nobusue H, Kano K. 2012. Dedifferentiated follicular granulosa cells derived from pig ovary can transdifferentiate into osteoblasts. Biochem J, 447:239-248.

Parte S, Bhartiya D, Telang J, Daithankar V, Salvi V, Zaveri K, Hinduja I. 2011. Detection, characterization, and spontaneous differentiation in vitro of very small embryonic-like putative stem cells in adult mammalian ovary. Stem Cells Dev, 20(8):1451-1464.

Potten CS, Loeffler M. 1990. Stem cells: attributes, cycles, spirals, pitfalls and uncertainties. Lessons for and from the crypt. Development, 110:1001-1020.

Rodgers RJ, Lavranos TC, van Wezel IL, IrvingRodgers HF. 1999. Development of the ovarian follicular epithelium. Mol Cell Endocrinol, 151(12):171-179.

Rodgers RJ, Irving-Rodgers HF, van Wezel IL, Krupa M, Lavranos TC. 2001. Dynamics of the membrana granulosa during expansion of the ovarian follicular antrum. Mol Cell Endocrinol, 171(1-2):41-48.

Sumer H, Liu J, Malaver-Ortega LF, Lim ML, Khodadadi K, Verma PJ. 2011. NANOG is a key factor for induction of pluripotency in bovine adult fibroblasts. J Anim Sci, 89:2708-2716.

Townson DH, Combelles CMH. 2012. Ovarian Follicular Atresia, Basic Gynecology. Some Related Issues. InTech, 44-76.

Varras M, Griva T, Kalles V, Akrivis C, Paparisteidis N. 2012. Markers of stem cells in human ovarian granulosa cells: is there a clinical significance in ART?. J Ovarian Res, 5:36. 